\title{
Fístula uretrocavernosa por fractura penena
}

\author{
Juaneda Castell B, Montlleó González M, Ponce de León Roca X, Gausa Gascón L1, \\ Caparrós Sariol J, Villavicencio Mavrich H.
}

Servicio de Urología. Fundació Puigvert. Barcelona.

Actas Urol Esp. 2008;32(10):1043-1045

\section{RESUMEN}

FÍSTULA URETROCAVERNOSA POR FRACTURA PENENA

La fractura de pene con laceración de la uretra se presenta con dolor y hematoma, detumescencia, fallo en la erección y uretrorragia. Describimos el tercer caso publicado en la literatura de fistula uretrocavernosa por fractura peneana ocurrida durante el coito.

Palabras clave: Fístula uretrocavernosa. Factura penena.

\section{ABSTRACT \\ URETHROCAVERNOUS FISTULA DUE TO PENILE FRACTURE}

Penile fracture with urethral laceration usually presents with pain and haematoma, detumescence, erectile failure and blood loss through urethral meatus. We describe the third published case of urethrocavernous fistula following blunt penile trauma sustained durind sexual intercourse.

Keywords: Urethrocavernous fistula. Penile fracture.

$\mathrm{L}$ a "fractura" peneana -rotura de los cuerpos cavernosos por traumatismo del pene erecto- es una entidad poco frecuente pero grave por las complicaciones que puede ocasionar. La causa de la rotura es el pinzamiento de la túnica albugínea, con la consiguiente elevación de la presión intracavernosa y rotura de la misma. En algunas ocasiones la rotura de los cuerpos cavernosos se asocia a lesión del cuerpo esponjoso uretral. La fractura del pene con laceración de la uretra se presenta no sólo con dolor, hematoma y detumescencia penena sino también con uretrorragia en la mayoría de los casos.

\section{CASO CLÍNICO}

Paciente varón de 37 años que acudió a urgencias a las 48 horas de sufrir un traumatismo peneano durante el coito. Refería uretrorragia y cierta dificultad miccional. En la exploración se observaba un mínimo hematoma en la base del pene y una zona dolorosa a la palpación a nivel del cuerpo cavernoso derecho. No se evidenciaba desviación peneana.
Se realizó ecografia que demostró solución de continuidad a nivel de tercio medio de la albuginea del cuerpo cavernoso derecho que abría comunicación entre éste y el cuerpo esponjoso, sin evidenciar colecciones.

Con la sospecha de fístula uretrocavernosa se realizó uretrografía retrógrada que demostró la presencia de una comunicación fistulosa a nivel de la porción medio peneana que comunica la uretra con el cuerpo cavernoso derecho (Fig. 1).

Se colocó sonda de cistostomía como derivación urinaria y se instauró tratamiento antibiótico. Tras 48 horas de observación se dio el alta hospitalaria al paciente con sonda de cistostomía.

En el control posterior al mes del traumatismo, el paciente refería erecciones de buena calidad equiparables a las erecciones previas al traumatismo, sin dolor, uretrorragia ni desviación peneana durante la erección. La uretrografía retrógrada realizada demostró la presencia de una ligera estenosis uretral anular localizada en la zona del traumatismo. (Fig. 2 y 3). La flujometría realizada evidenció buen flujo urinario (Q máx: $17 \mathrm{ml} / \mathrm{seg}$, Volumen $167 \mathrm{cc}$ ) y ausencia de residuo postmiccional. 


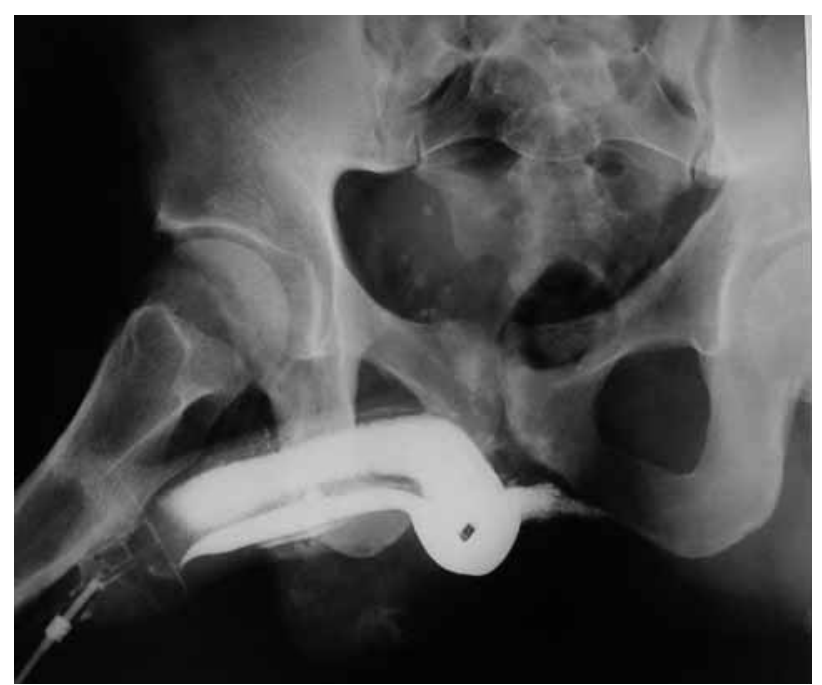

FIGURA 1. Uretrografia retrógrada: Presencia de la fistula uretrocavernosa y llenado retrógrado del cuerpo cavernoso.

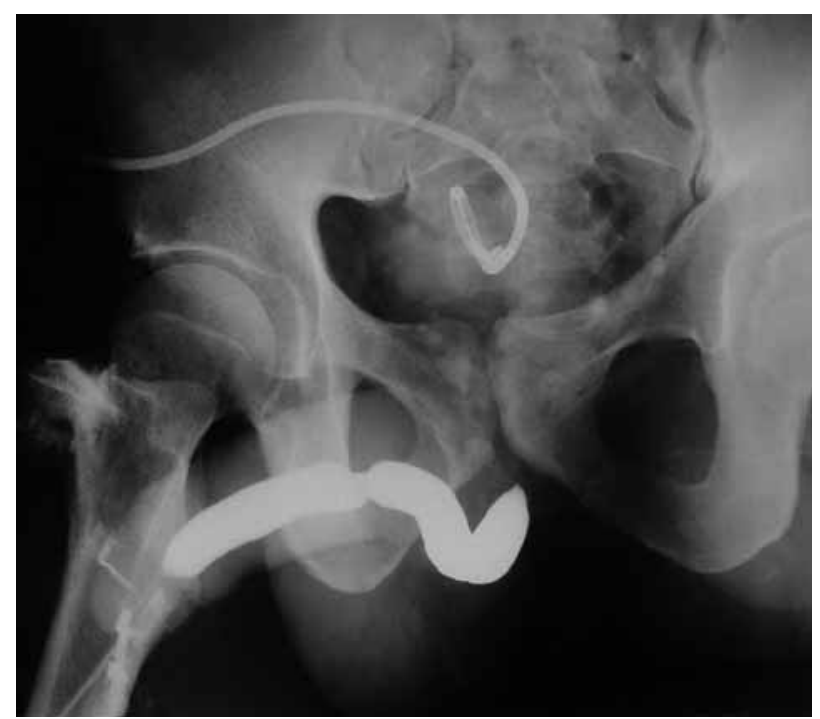

FIGURA 2. Uretrografia retrógrada al mes del traumatismo peneano: Presencia de un pequeño anillo estenótico a nivel medio peneano.

\section{DISCUSION}

La historia natural de la fractura pene es clásica: Una torcedura en el pene erecto acompañado de un chasquido, dolor e inmediata reversión de la tumescencia.

El examen físico puede mostrar edema y equimosis. El defecto de la albugínea casi siempre es palpable $^{1}$, pero puede estar enmascarado por la inflamación y el hematoma.

Uno de los motivos por los cuales durante la rigidez penene aumenta el riesgo de fractura es que la túnica albugínea se elonga y se afina significativa-

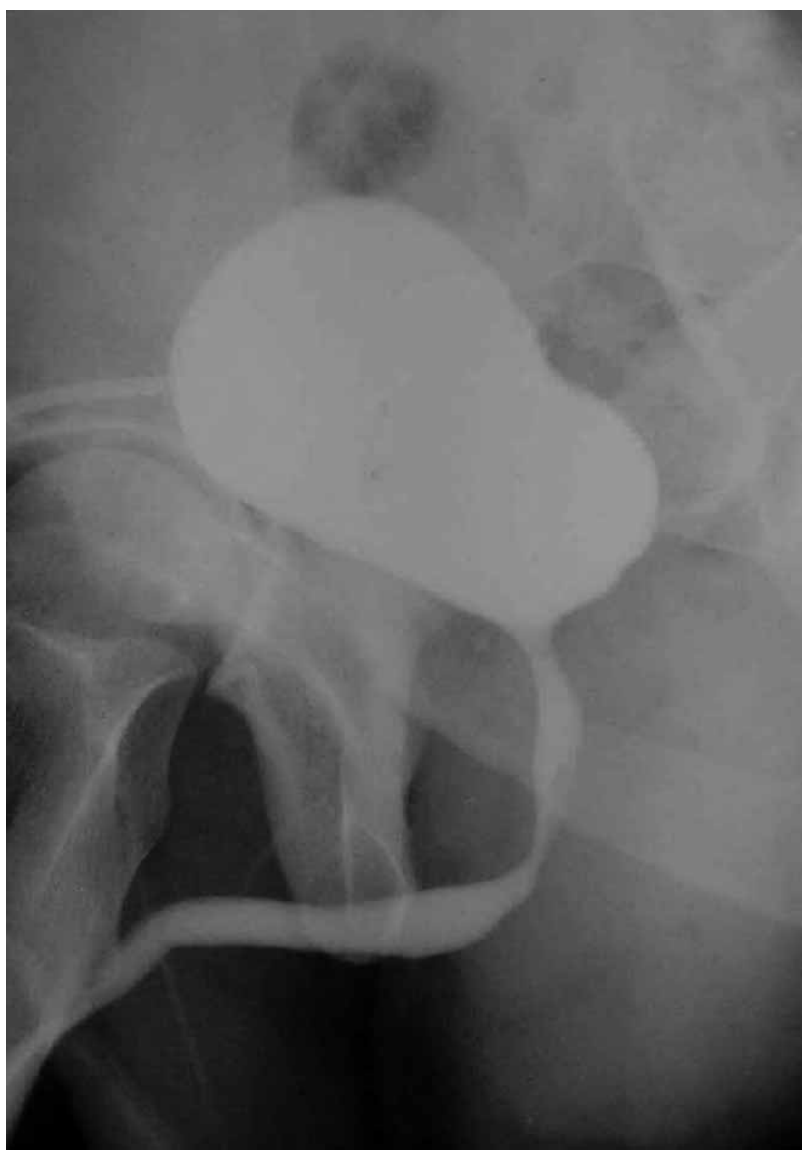

FIGURA 3. Uretrografia miccional al mes del traumatismo peneano.

mente durante la erección; en estado de flaccidez tiene un grosor máximo de $2,4 \mathrm{~mm}$ y durante la erección su espesor puede ser tan fino como de 0,25 $\mathrm{mm}$ a $0,5 \mathrm{~mm}^{2}$.

La fractura de pene está asociada con lesión uretral entre el 10 y $33 \%$ de los casos según las series ${ }^{3}$. Hay que sospechar dicha lesión en presencia de uretrorragia, disuria o retención aguda de orina. La uretrografía debe realizarse en los casos de sospecha de lesión uretral asociada ${ }^{4,5}$.

Se ha empleado la ecografía para el diagnóstico de las fracturas peneanas, aunque en la mayoría de los casos el cuadro clínico es suficientemente claro, por lo que se puede prescindir de ella.

La cavernosografía y la RMN pueden ser útiles en pacientes con presentación atípica, para confirmar el diagnóstico antes de la cirugía.

La mayoría de los autores recomiendan una exploración quirúrgica inmediata en las primeras 36 horas ya que así las secuelas son mínimas, comparado con la alta incidencia (10-30\%) de desviación peneana si el tratamiento es diferido ${ }^{6-8}$. 
El tratamiento quirúrgico consiste en la evacuación del hematoma, control de la hemorragia y reparación de la solución de continuidad de los cuerpos cavernosos y lesiones uretrales asociadas. La incisión circunferencial distal, a nivel del surco balano prepucial, con la realización del "degloving” peneano, permite una correcta exposición de los cuerpos cavernosos y de la uretra peneana para la reparación de la fractura ${ }^{9}$.

Si existe sección uretral importante, se debe realizar una buena disección uretral para su movilización, la cual permita después una correcta anastomosis sin tensión ${ }^{10}$.

Si hay lesión uretral y se opta por un tratamiento diferido, siempre hay que drenar la orina mediante sonda de cistostomía, y a partir de los tres meses, cuando el tejido uretral ha cicatrizado y es maduro, puede llevarse a cabo la reconstrucción quirúrgica previa actualización de los estudios de imagen.

En la literatura tan solo hay dos casos descritos de fístula uretrocavernosa por traumatismo peneano. El primer caso fue descrito por Hargreaves en 1994: El paciente presentaba una laceración uretral de 3 cm diagnosticada por uretroscopia, lo cual requirió reparación quirúrgica urgente. El segundo caso fue descrito por Hanif en 1999: la fistula uretrocavernosa fue diagnosticada por cavernosografía, ésta era de pequeña magnitud por lo que se optó por realizar derivación urinaria suprapúbica y conducta expectante. La evolución de ambos pacientes fue buena: no desarrollaron estenosis de uretra y mantuvieron erecciones correctas sin desviación peneana ${ }^{11,12}$.

En nuestro caso se optó por realizar un tratamiento conservador ya que el paciente acudió a nuestro centro a las 48 horas de haber sufrido el traumatismo y en ese momento no presentaba uretrorragia ni signos de sangrado activo.

\section{REFERENCIAS}

1. Cendron M, Whitmore KE, Carpiniello V, Kurzweil SJ, Hanno PM, Snyder HM, et al. Traumatic rupture of the corpus cavernosum: evaluation and management. J Urol. 1990;144(4):987991.

2. Bitsch M, Kromann-Andersen B, Schou J, Sjontoft E. Elasticity and the tensile strength of tunica albuginea of the corroa cavernosa. J Urol. 1990;143(3):642-645.

3. Kowalczy J, Athens A, Grimaldi A. Penile fractura: an unusual presentation with lacerations of bilateral corposa cavernosa and parcial disruption of urethra. Urology 1994;145:12671270.

4. Fergany AF, Angermeier KW, Montague DK. Review of Cleveland Clinic experience with penile fracture. Urology. 1999;54(2):352355.

5. Zargooshi J. Penile fracture in Kermanshah, Iran: Reposrt of 172 cases. J Urol. 2000;164(2):364-366.

6. Kibria SA, Islam MF, Bhuyan ZI. Fracture penis- a study of twenty cases. Bangladesh Med Res Counc Bull. 2001;27(1):3337.

7. Karadeniz T, Topsakal M, Ariman A, Erton H, Basak D. Penile fracture: differential diagnosis, management and outcome. $\mathrm{Br}$ J Urol. 1996;77(2):279-281.

8. Asgari MA, Hosseini SY, Safarinejad MR, Samadzadeh B, Bardideh AR. Penile fractures: Evaluation, therapeutic approaches ang lon-term results. J Urol. 1996;155(1):148-149.

9. Mydlo JH. Surgeon esperience with penile fracture. J Urol. 2001;166(2):526-529.

10. Devine CW Jr, Jordan GH, Schlossberg SM. Surgery of the penis and uretra. In walsh PC, Retik AB, Vaughan ED, Wein AJ eds. Campbell's Urology. $6^{\text {th }}$ edn. Philadelphia: WB Saunders, 1992:2957-3032.

11. Hargreaves DG, Plail RO. Fracture of the penis causing a corporo-urethral fistula. Br J Urol. 1994;73(1):97.

12. Motiwala HG. Urethrocavernous fistula following sexual intercourse. J Urology. 1999;149(2):371.

Correspondencia autor: Dra. B Juaneda Castell

Servicio de Urología. Fundació Puigvert

Cartagena, 340-350 - 08025 Barcelona

Tel.: 934169700

E-mail autor: bjuaneda73@hotmail.com

Trabajo recibido: abril 2007

Trabajo aceptado: julio 2007 